\title{
Metastatic Melanoma to the Pancreas Forming Portal Vein Tumor Thrombosis: A Case Report
}

\author{
Dong Kyun Kim, ${ }^{1}$ Joon-Il Choi, ${ }^{1,}{ }^{*}$ Moon Hyung Choi, ${ }^{1}$ Chandana Lall, ${ }^{2}$ Eun Sun Jung, ${ }^{3}$ and In Seok Lee ${ }^{4}$ \\ ${ }^{1}$ Department of Radiology, Seoul St. Mary's Hospital, College of Medicine, the Catholic University of Korea, 222 Banpo-daero, Seocho-gu, Seoul, 06591, Republic of Korea \\ ${ }^{2}$ Department of Radiological Sciences, University of California, Irvine, 101 The City Drive South, Orange, CA 92868, the Unites States of America \\ ${ }^{3}$ Department of Hospital Pathology, Seoul St. Mary's Hospital, College of Medicine, the Catholic University of Korea, 222 Banpo-daero, Seocho-gu, Seoul, 06591, Republic of \\ Korea \\ ${ }^{4}$ Division of Gastroenterology, Department of Internal Medicine, Seoul St. Mary's Hospital, College of Medicine, the Catholic University of Korea, 222 Banpo-daero, Seocho-gu, \\ Seoul, 06591, Republic of Korea
}

"Corresponding author: Joon-Il Choi, Department of Radiology, Seoul St. Mary's Hospital, College of Medicine, The Catholic University of Korea, 222 Banpo-daero, Seocho-gu, Seoul 06591, Republic of Korea. Tel: +82-222581431, Fax:+82-25996771, E-mail: dumkycji@gmail.com

Received 2016 January 12; Revised 2016 July 31; Accepted 2016 September 25.

\begin{abstract}
Pancreatic metastasis of malignant melanoma is rare and porto-splenic tumor thrombus associated with malignant melanoma is even less frequent. We report a case of metastatic malignant melanoma involving the pancreas, portal vein, splenic vein and superior mesenteric vein. A 60-year-old man showed an elevated amylase level. He had a history of sigmoid colon adenocarcinoma and malignant melanoma. Computed tomography (CT) and magnetic resonance imaging(MRI) showed extensive thrombosis involving the intra- and extrahepatic portal veins extending into the superior/inferior mesenteric vein and splenic vein. On T1 weighted images, tumor thrombi demonstrated diffusely high signal intensity, which is a characteristic finding in malignant melanoma. Also, thrombus showed prominent diffusion restriction on diffusion weighted images and intense uptake of 18F-fluorodeoxyglucose (18FFDG) on positron emission tomography (PET). Ultrasound-guided biopsy confirmed the metastatic, malignant melanoma involving the pancreas and the veins. We report an unusual manifestation of metastatic, malignant melanoma to the pancreas with extensive tumor thrombi. Specific MR features including high signal intensity on T1 weighted images and diffusion restriction on diffusion weighted images were helpful in reaching a conclusion.
\end{abstract}

Keywords: Malignant Melanoma, Magnetic Resonance Imaging, Pancreas, Tumor Thrombus

\section{Introduction}

Pancreas is a favored site for metastases from primary tumors of the lung, kidney and gastrointestinal (GI) tract although metastases from other primary tumors have been reported (1-3). Metastatic melanomas to the pancreas have been reported very rarely and there is only a singular case report on portal vein thrombus formation by metastatic pancreatic melanoma. Also, there was no report dealing with the imaging features of this exceptional rare condition (4). We report a case of metastatic melanoma to the pancreas with extensive tumor thrombosis in intra- and extrahepatic portal veins, the splenic vein, hepatic veins and right atrium. Magnetic resonance (MR) imaging features were helpful in differentiation of metastatic melanoma from other neoplastic entities. The institutional review board approved the report of this case.

\section{Case Presentation}

A 60-year-old man was referred to the hospital for work-up of abdominal pain and dyspepsia. He had a history of sigmoid colon adenocarcinoma and underwent surgical resection of the colonic mass and hepatic metastatectomy (wedge resection) 14 years ago. Peritoneal carcinomatosis was diagnosed four years ago and the patient underwent several cycles of chemotherapy.

The patient was also diagnosed with malignant melanoma of the left foot 10 years ago and underwent surgical resection of a pulmonary metastasis of malignant melanoma three years ago. The laboratory data revealed an elevated amylase (313.0 U/L) and lipase level (534.7 U/L) raising the possibility of acute pancreatitis.

Computed tomography (CT) of the abdomen showed extensive thrombosis of the intra- and extrahepatic portal vein, hepatic vein, right atrium, superior and inferior mesenteric vein, and splenic vein (Figure 1A). Magnetic resonance (MR) imaging of the pancreas was performed and T2 weighted images revealed an ill-defined mass involving the head of the pancreas (Figure $1 \mathrm{~B}$ ) and extensive tumor thrombus in the portal and splenic veins. On T1 weighed images, high signal intensity of the tumor and venous thrombus was noted (Figure 1C and D). Thrombus showed high signal intensity, which means diffusion restriction on diffusion-weighted images (Figure 1E). Apparent diffu- 
sion coefficient (ADC) values of tumor thrombus of the intrahepatic portal vein, splenic veins, and pancreatic mass were $0.985 \times 10^{-3} \mathrm{~mm}^{2} / \mathrm{s}, 0.876 \times 10^{-3} \mathrm{~mm}^{2} / \mathrm{s}$, and $0.923 \times$ $10^{-3} \mathrm{~mm}^{2} / \mathrm{s}$, respectively. 18F-fluorodeoxyglucose (18F-FDG) positron emission tomography (PET) showed intense ${ }^{18} \mathrm{~F}$ FDG uptake within the tumor thrombi (Figure 1F).

An ultrasound-guided biopsy of the pancreatic head mass was performed to make a definitive diagnosis for selection of appropriate chemotherapy. On ultrasonography, the mass was ill defined and hypoechoic at the pancreatic head. A gross specimen by needle biopsy showed brownish tissue. Fontana Masson staining for detecting melanin pigments, human melanoma black 45 (HMB-45) staining for diagnosing melanoma and S-100 protein staining which can be positive in the specimen of melanoma, schwannomas, neurofibromas and malignant peripheral nerve sheath tumors were performed. Positive staining for all three markers was demonstrated and the pathological diagnosis of malignant melanoma was confirmed (Figure $1 G)$.

\section{Discussion}

Metastases to the pancreas are relatively uncommon; however, not rare. In a large series of surgical specimens and autopsies, the prevalence of metastatic, secondary tumors of the pancreas is approximately $2-4 \%(3,5)$. In a patient with a known primary tumor, the possibility of metastasis should be considered over a primary pancreatic cancer. Not only do primary tumors of the lung, kidney, breast, and GI tract frequently metastasize to the pancreas, but also a variety of primary tumors originating from other sites including sarcomas, lymphoma, and ovarian cancers metastasize to the pancreas.

Malignant melanoma can also metastasize to the pancreas although relatively uncommon. A primary melanoma involving the pancreas, however, is extremely rare and the majority of melanomas involving the pancreas tend to be metastatic (4). Frequently, metastatic tumors are clinically misdiagnosed as primary pancreatic tumors (3). Moreover, it is quite challenging to clinically differentiate the primary origins of metastatic tumors involving the pancreas. In our case, besides melanoma, the patient had colon cancer with peritoneal carcinomatosis, which may confound the diagnosis.

In our case, MR imaging was helpful in diagnosing the tumor origin and thrombus characterization. First, restricted diffusion (high signal intensity on diffusion weighted images and decreased ADC values) noted in the splenic and portal veins implied malignant tumor thrombi (6). Second, high signal intensity of tumor thrombus on $\mathrm{T} 1$ weighted image was helpful for narrowing the differential diagnosis. In contrast to $\mathrm{T} 1$ hypointense lesions usually demonstrated in pancreas metastases from other primary cancers, melanoma has a typical high signal intensity on T1 weighted images due to melanin and iron secondary to hemorrhage with paramagnetic T1 shortening effect (7-9). About 50\% of melanomas show high signal intensity on T1 weighted images but this finding is rare in other malignancies (10). Non-tumorous, bland thrombus can be shown as high signal intensity on $\mathrm{T} 1$ weighted images, but in this case, intense 18F-FDG uptake in thrombi was clearly visible on PET and the diagnosis of tumor thrombus by malignant melanoma could be made.

This case highlights the characteristic MR appearance of metastatic malignant melanoma easily differentiated from other metastatic and primary lesions. Furthermore, MR appearance may be helpful in differentiating tumor thrombus from bland (non-neoplastic) thrombosis.

The etiology of high signal intensity of melanoma on T1 weighted images is controversial. Some authors emphasize the role of melanin itself as a paramagnetic material. Other authors emphasize the significance of blood products such as iron (11-13). Melanin and iron are paramagnetic materials and can also produce $\mathrm{T} 2^{*}$ weighted signal loss due to susceptibility effect (11) and T2* weighted images are reported to be helpful in melanoma characterization. However, in our case, we did not acquire T2* weighted images because it is not a standard sequence in abdominal MR imaging.

When it is difficult to differentiate pancreatic tumors due to similarities on imaging findings, it is reported that endoscopic ultrasound (EUS) with fine needle aspiration (FNA) often plays an important role in providing conclusive histopathological confirmation (14). In our case, we performed percutaneous biopsy because percutaneous approach to pancreas head mass, not to tumor thrombi, was relatively easy.

Symptoms from pancreatic neoplasms commonly include pain, weight loss, obstructive jaundice, and gastrointestinal bleeding (2). Less commonly, acute pancreatitis can occur, probably related to pancreatic duct obstruction by the tumor. Acute pancreatitis by malignant melanoma has been previously described (15). Likewise, in our case, the patient showed elevated amylase and lipase levels although he had no other abdominal symptoms (4). In our case, initial diagnosis of malignant melanoma was made 10 years ago and metastatectomy for a pulmonary mass was performed 3 years ago. A case of pancreatic metastasis of malignant melanoma with tumor thrombi was reported 15 years after initial surgery, but imaging features were not reported in that case (4).

Management of pancreatic metastasis from malignant melanoma is controversial. There is limited experience 

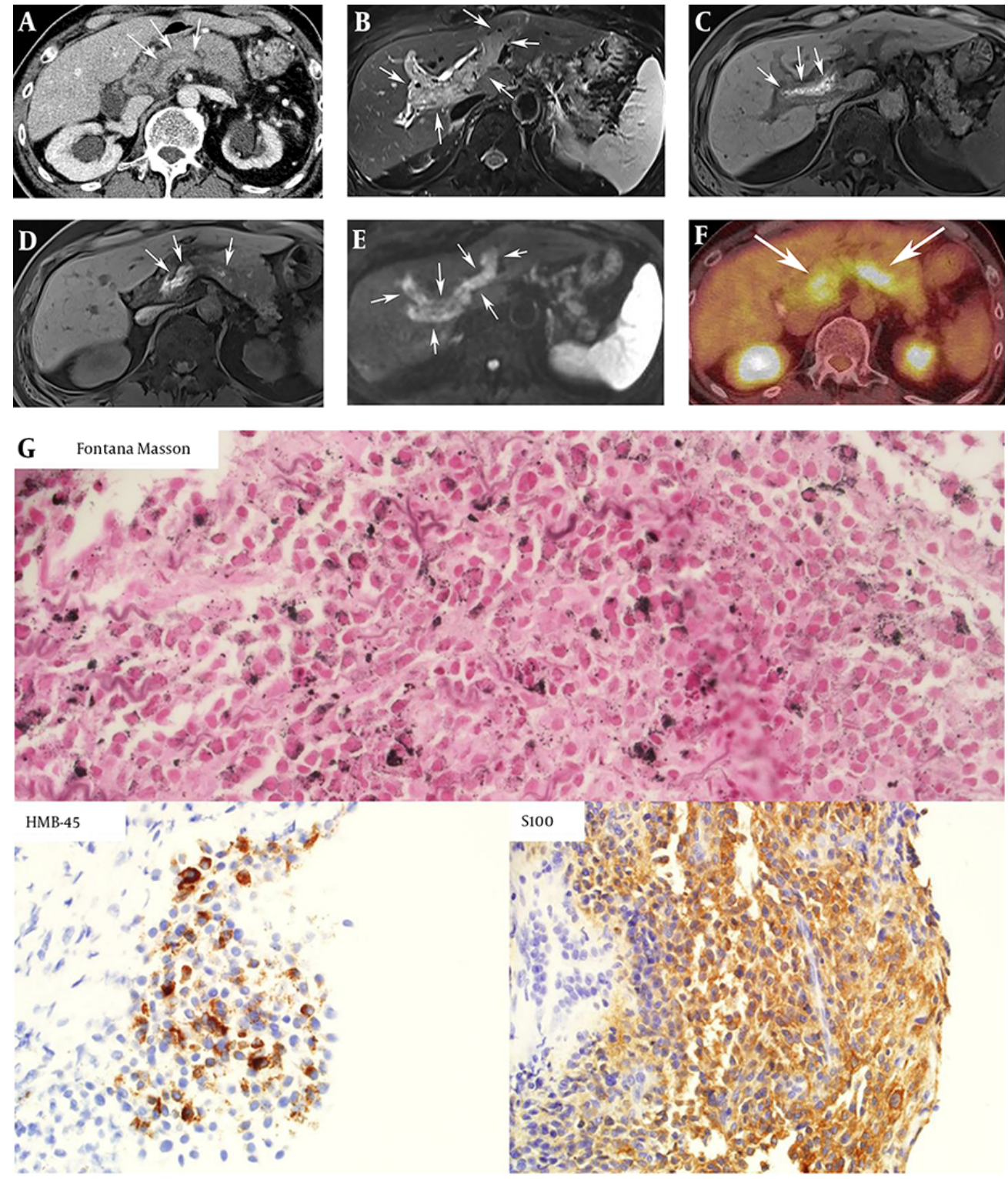

Figure 1. A 60-year-old man with metastatic melanoma to the pancreas with extensive vascular invasion. A, Axial CT scan of the abdomen shows diffuse tumor thrombosis involving splenic veins (arrows). Bilateral hydronephrosis is also noted; B, On T2 weighted image, extensive tumor thrombosis by malignant melanoma involving intrahepatic portal veins is noted (arrow); C, On T1 weighted image, tumor thrombi in portal veins show high signal intensities, which is the striking radiographic feature of malignant melanoma (arrow); D, Tumor thrombi in the splenic vein also show high signal intensity of T1 weighted image (arrows); E, Extensive thrombi in portal veins exhibit diffusion restriction (arrows) on diffusion weighted imaging with b values of $500 \mathrm{sec} / \mathrm{mm}^{2}$. Restricted diffusion of these thrombi highly suggests that the lesions are tumor thrombosis, rather than bland thrombosis; F, 18F-fluorodeoxyglucose positron emission tomography (18F-FDG-PET) shows intense FDG uptake in tumor thrombus in portal and splenic veins (arrows); G, Immunohistochemistry staining for Fontana Masson, which is a marker for melanin pigment (dark dots), HMB-45 for melanoma (brown color), and S-100 (brown color) all showed positive results, and the final diagnosis was metastatic melanoma.

comparing surgical resection of isolated metastasis versus non-surgical management and the 5-year survival (16, 17). A larger cohort and well-designed study is necessary to confirm the efficiency of surgical treatment of isolated metastatic lesions in the pancreas $(18,19)$. In our case, the patient was neither a surgical nor chemotherapy candi- date due to concurrent, disseminated colon cancer and extensive tumor thrombosis by malignant melanoma. In addition, the performance of the patient was poor; the Eastern cooperative oncology group (ECOG) score was 3.

In conclusion, we report the unusual manifestation of metastatic, malignant melanoma to the pancreas with 
extensive tumor thrombi. Specific MR features including high signal intensity on T1 weighted images and diffusion restriction on diffusion weighted images were helpful in reaching a conclusion.

\section{Footnotes}

\section{Authors' Contribution: None declared. Financial Disclosure: None declared. Funding/Support: None declared.}

\section{References}

1. Roland CF, van Heerden JA. Nonpancreatic primary tumors with metastasis to the pancreas. Surg Gynecol Obstet. 1989;168(4):345-7. [PubMed: 2928909].

2. Z'Graggen K, Fernandez-del Castillo C, Rattner DW, Sigala H, Warshaw AL. Metastases to the pancreas and their surgical extirpation. Arch Surg. 1998;133(4):413-7. [PubMed: 9565122].

3. Adsay NV, Andea A, Basturk O, Kilinc N, Nassar H, Cheng JD. Secondary tumors of the pancreas: an analysis of a surgical and autopsy database and review of the literature.Virchows Arch. 2004;444(6):52735. doi: 10.1007/s00428-004-0987-3. [PubMed: 15057558].

4. Lee S, Choi JI, Park MY, Yeo DM, Byun JY, Jung SE, et al. Intra- and interobserver reliability of gray scale/dynamic range evaluation of ultrasonography using a standardized phantom. Ultrasonography. 2014;33(2):91-7. doi: 10.14366/usg.13021. [PubMed: 24936501].

5. Reddy S, Wolfgang CL. The role of surgery in the management of isolated metastases to the pancreas. The Lancet Oncology. 2009;10(3):28793. doi: 10.1016/s1470-2045(09)70065-8.

6. Catalano OA, Choy G, Zhu A, Hahn PF, Sahani DV. Differentiation of malignant thrombus from bland thrombus of the portal vein in patients with hepatocellular carcinoma: application of diffusionweighted MR imaging. Radiology. 2010;254(1):154-62. doi: 10.1148/radiol.09090304. [PubMed: 20032150].

7. Merkle EM, Boaz T, Kolokythas O, Haaga JR, Lewin IS, Brambs HJ Metastases to the pancreas. Br J Radiol. 1998;71(851):1208-14. doi: 10.1259/bjr.71.851.10434919. [PubMed: 10434919].
8. Balci NC, Semelka RC. Radiologic features of cystic, endocrine and other pancreatic neoplasms. Euro J Radiol. 2001;38(2):113-9. doi 10.1016/s0720-048x(01)00296-0.

9. Premkumar A, Marincola F, Taubenberger J, Chow C, Venzon D, Schwartzentruber D. Metastatic melanoma: correlation of MRI characteristics and histopathology. J Magn Reson Imaging. 1996;6(1):190-4 [PubMed: 8851427].

10. Shindoh N, Ozaki Y, Kyogoku S, Nakanishi A, Sumi Y, Katayama H Osteoclast-type giant cell tumor of the pancreas: helical CT scans. AJR Am J Roentgenol. 1998;170(3):653-4. doi: 10.2214/ajr.170.3.9490947. [PubMed: 9490947].

11. Ichikawa T, Federle MP, Ohba S, Ohtomo K, Sugiyama A, Fujimoto $H$, et al. Atypical exocrine and endocrine pancreatic tumors (anaplastic, small cell, and giant cell types): CT and pathologic features in 14 patients. Abdom Imaging. 2000;25(4):409-19. [PubMed:10926196].

12. Leighton CC, Shum DT. Osteoclastic Giant Cell Tumor of the Pancreas. American J Clinical Oncol : Cancer Clinical Trials. 2001;24(1):77-80. doi: 10.1097/00000421-200102000-00014.

13. van Rhijn BD, Warners MJ, Curvers WL, van Lent AU, Bekkali NL, Takkenberg RB, et al. Evaluating the endoscopic reference score for eosinophilic esophagitis: moderate to substantial intra- and interobserver reliability. Endoscopy. 2014;46(12):1049-55. doi: 10.1055/s-0034 1377781. [PubMed: 25208033].

14. Jana T, Caraway NP, Irisawa A, Bhutani MS. Multiple pancreatic metastases from malignant melanoma: Conclusive diagnosis with endoscopic ultrasound-guided fine needle aspiration. Endosc Ultrasound 2015;4(2):145-8. doi: 10.4103/2303-9027.156746. [PubMed: 26020050].

15. Sobesky R, Duclos-Vallee JC, Prat F, Pelletier G, Encaoua R, Boige V, et al. Acute pancreatitis revealing diffuse infiltration of the pancreas by melanoma. Pancreas. 1997;15(2):213-5. [PubMed: 9260209].

16. Fletcher WS, Pommier RF, Lum S, Wilmarth TJ. Surgical treatment of metastatic melanoma. America J Surg. 1998;175(5):413-7. doi: 10.1016/s0002-9610(98)00041-5.

17. Bruix J, Sherman M, American Association for the Study of Liver Diseases . Management of hepatocellular carcinoma: an update. Hepatol ogy. 2011;53(3):1020-2. doi: 10.1002/hep.24199. [PubMed: 21374666].

18. Goyal J, Lipson EJ, Rezaee N, Edil BH, Schulick R, Wolfgang CL, et al. Surgical resection of malignant melanoma metastatic to the pancreas: case series and review of literature. J Gastrointest Cancer. 2012;43(3):431-6. doi: 10.1007/s12029-011-9320-y. [PubMed: 21912850].

19. Birnbaum DJ, Moutardier V, Turrini O, Goncalves A, Delpero JR. Isolated pancreatic metastasis from malignant melanoma: is pan createctomy worthwile?. J Surg Tech Case Rep. 2013;5(2):82-4. doi: 10.4103/2006-8808.128733. [PubMed: 24741425] 